\title{
A Unified Approach to Measurement Error and Missing Data: Details and Extensions
}

Sociological Methods \& Research 2017, Vol. 46(3) 342-369

(C) The Author(s) 2015

Reprints and permission: sagepub.com/journalsPermissions.nav DOI: $10.1177 / 0049124115589052$ journals.sagepub.com/home/smr

\section{Matthew Blackwell', James Honaker', and Gary King'}

\begin{abstract}
We extend a unified and easy-to-use approach to measurement error and missing data. In our companion article, Blackwell, Honaker, and King give an intuitive overview of the new technique, along with practical suggestions and empirical applications. Here, we offer more precise technical details, more sophisticated measurement error model specifications and estimation procedures, and analyses to assess the approach's robustness to correlated measurement errors and to errors in categorical variables. These results support using the technique to reduce bias and increase efficiency in a wide variety of empirical research.
\end{abstract}

\section{Keywords}

missing data, measurement error, imputation, multiple overimputation

\section{Introduction}

In this article, we extend and technically undergird a new unified approach to analyses of data sets with both measurement error and missing data,

\footnotetext{
' Institute for Quantitative Social Science, Harvard University, Cambridge, MA, USA

Corresponding Author:

Gary King, Institute for Quantitative Social Science, Harvard University, 1737 Cambridge Street, Cambridge, MA 02138, USA.

Email: king@harvard.edu
} 
extremely common coexisting conditions throughout applied social science research. The technique is called multiple overimputation (MO) and it generalizes the popular approach of multiple imputation (MI) for missing data as well as a methodology for measurement error. The technique benefits by formalizing the idea of regarding missing data as an extreme form of measurement error, or measurement error as a form of partially missing data. Applied researchers can easily use the technique to preprocess their data to deal with both methodological problems simultaneously and then use whatever technique they might have applied to their data if it had neither problem.

Blackwell, Honaker, and King (2017; BHK, hereinafter) offer an overview of MO, along with empirical applications and suggestions for practice. In this article, we first offer a more precise formulation of the methodology, its assumptions, and its implementation (second section). Next, we show how to estimate rather than assume the variances in more sophisticated measurement error models (third section). We then show how MO remains robust for common categorical variables even when specifying a model for continuous measurement error (fourth section) and for different types of correlated measurement error when the measurement error model assumes independence (fifth section). Finally, we offer detailed simulations to provide guidance for the number of data sets to overimpute (sixth section). The seventh section concludes.

\section{Model and Estimation}

Here we introduce the general MO model and a specific expectation-maximization (EM) algorithm implementation. We also show that it is equivalent to MI with observation-level priors as introduced by Honaker and King (2010). We also offer more general notation than that in BHK.

\section{Notation}

Consider data $x_{i j}$ with observations $i=1, \ldots, N$ and variables $j=1, \ldots, p$. Although numerical values for all elements of $x \equiv\left\{x_{i j} \mid \forall i, j\right\}$ exist, we have knowledge about each through a three-part measurement mechanism:

$$
m_{i j}=\left\{\begin{array}{l}
0 \text { if } x_{i j} \text { is observed } \\
1 \text { if } x_{i j} \text { is missing and an unbiased proxy } w_{i j} \text { is observed } \\
2 \text { if } x_{i j} \text { is missing }
\end{array}\right.
$$

The three cases correspond respectively to $(0)$ direct observation of the true value; (1) unbiased estimation of the true value, $E\left(w_{i j}\right)=x_{i j}$ and 
(2) classical missingness, for which the true value and proxies of it are unavailable. We partition $x$ into sets where the true values are observed or missing: $x=\left\{x_{\mathrm{obs}}, x_{\mathrm{mis}}\right\}$, where

$$
\begin{gathered}
x_{\mathrm{obs}} \equiv\left\{x_{i j} \mid(\forall i, j) \wedge\left(m_{i j}=0\right)\right\} \quad \text { and } \\
x_{\text {mis }} \equiv\left\{x_{i j} \mid(\forall i, j) \wedge\left(m_{i j}>0\right)\right\} .
\end{gathered}
$$

Thus, we observe portions of the covariates $x_{\text {obs }}$, the measurement mechanism $m \equiv\left\{m_{i j} \mid \forall i, j\right\}$, and the proxies $w \equiv\left\{w_{i j} \mid(\forall i, j) \wedge\left(m_{i j}=1\right)\right\}$.

Our goal is to infer the distribution of $x, p(x \mid \theta) \equiv p\left(x_{\mathrm{obs}}, x_{\mathrm{mis}} \mid \theta\right)$, where $\theta$ is an unknown parameter vector. We begin with the observed-data probability density function

$$
\begin{aligned}
p\left(m, w, x_{\mathrm{obs}} \mid \theta, \gamma, \phi\right)=\int & p\left(m \mid x_{\mathrm{obs}}, x_{\mathrm{mis}}, w, \phi\right) \\
& \times p\left(w \mid x_{\mathrm{mis}}, x_{\mathrm{obs}}, \gamma\right) p\left(x_{\mathrm{obs}}, x_{\mathrm{mis}} \mid \theta\right) \mathrm{d} x_{\mathrm{mis}},
\end{aligned}
$$

where $\phi$ is the parameter of the distribution of $m$, and $\gamma$ is the parameter of the distribution of the measurement mechanism.

\section{Assumptions}

We require two key assumptions. First, we enable researchers to avoid specifying a full probability density for the measurement assignment, $p\left(m \mid x_{\mathrm{obs}}\right.$, $\left.x_{\text {mis }}, w, \phi\right)$. To do this, we generalize MI's (realized) missing at random assumption:

\section{Assumption 1 (Ignorable Measurement Mechanism Assignment}

IMMA): (a) For any values of $m, x_{o b s}, w, x_{m i s}$, and $x_{m i s}^{\prime}$,

$$
p\left(m \mid x_{o b s}, x_{m i s}, w, \phi\right)=p\left(m \mid x_{o b s}, x_{m i s}^{\prime}, w, \phi\right) ;
$$

and (b) the parameters governing $m, \phi$, are distinct from those governing $w, \gamma$, and $x, \theta$, in equation (4).

Here, $x_{\mathrm{mis}}$ and $x_{\mathrm{mis}}^{\prime}$ are two possible realizations of the missing data, so that equation (5) says that the distribution of the mismeasurement indicator, $m$, is the same no matter the value of the missing data.

We also assume knowledge of the measurement error data generation process, allowing its indexed parameter vector to be known or estimated. 
Assumption 2 (Measurement error distribution). The distribution $p\left(w \mid x_{m i s}, x_{o b s}, \gamma\right)$ is known up to its parameters $\gamma$. The parameters $\gamma$ are either known or a consistent estimator $(\hat{\gamma}$ s.t. $\hat{\gamma} \stackrel{p}{\longrightarrow} \gamma)$ is available.

\section{The Observed-data Likelihood}

We begin with three definitions. First, we specify the joint likelihood that explicitly handles the presence of missingness or measurement error:

$$
L_{j}(\theta, \gamma, \phi)=p\left(m, w, x_{\mathrm{obs}} \mid \theta, \gamma, \phi\right) .
$$

Second, we write the profile likelihood of $(\theta, \gamma)$ with respect to the parameters of $m$ :

$$
L_{p}(\theta, \gamma)=\max _{\phi}\left[L_{j}(\theta, \gamma, \phi)\right] .
$$

The profile likelihood is useful because the maximum likelihood estimates (MLEs) from it will turn out to be the MLEs based on the joint likelihood. Finally, we define the ignorance likelihood, ignoring the measurement indicator $m$ :

$$
L(\theta, \gamma)=p\left(w, x_{\mathrm{obs}} \mid \theta, \gamma\right) .
$$

We now use these definitions to establish the relationship between these quantities, in a manner similar to results for the missingness at random assumption in classical analyses of MI for missing data.

Theorem 1: Under IMMA (Assumption 1), the profile likelihood for $(\theta, \gamma)$ is proportional to the ignorance likelihood: $L_{p}(\theta, \gamma) \propto L(\theta, \gamma)$.

Proof. Under IMMA, we can factor the joint likelihood as

$$
\begin{aligned}
L_{j}(\theta, \gamma, \phi) & =p\left(m, w, x_{\mathrm{obs}} \mid \theta, \gamma, \phi\right) \\
& =\int p\left(x_{\mathrm{obs}}, x_{\mathrm{mis}} \mid \theta\right) p\left(w \mid x_{\mathrm{mis}}, x_{\mathrm{obs}}, \gamma\right) p\left(m \mid x_{\mathrm{obs}}, x_{\mathrm{mis}}, w, \phi\right) d x_{\mathrm{mis}}, \\
& =\int p\left(x_{\mathrm{obs}}, x_{\mathrm{mis}} \mid \theta\right) p\left(w \mid x_{\mathrm{mis}}, x_{\mathrm{obs}}, \gamma\right) p\left(m \mid x_{\mathrm{obs}}, w, \phi\right) d x_{\mathrm{mis}}, \\
& =p\left(m \mid x_{\mathrm{obs}}, w, \phi\right) \int p\left(x_{\mathrm{obs}}, x_{\mathrm{mis}} \mid \theta\right) p\left(w \mid x_{\mathrm{mis}}, x_{\mathrm{obs}}, \gamma\right) d x_{\mathrm{mis}}, \\
& =p\left(m \mid x_{\mathrm{obs}}, w, \phi\right) L(\theta, \gamma) .
\end{aligned}
$$


The second equality is simply the definition of the joint likelihood, the third follows from IMMA, and the fourth and fifth from the properties of expectations. Substituting this expression into the definition of the profile likelihood (equation (7)) gives

$$
\begin{aligned}
& L_{p}(\theta, \gamma)=\max _{\phi}\left[p\left(m \mid x_{\mathrm{obs}}, w, \phi\right) L(\theta, \gamma)\right] \\
& =L_{p}(\theta, \gamma) \max _{\phi}\left[p\left(m \mid x_{\mathrm{obs}}, w, \phi\right)\right] \\
& \propto L(\theta, \gamma),
\end{aligned}
$$

where the last equality holds because the second factor of the second line is constant with respect to $(\theta, \gamma)$.

What Theorem 1 tells us is that inferences based on $L(\theta, \gamma)$ will be the same as inferences about $\theta$ and $\gamma$ from the profile likelihood and, thus, the joint likelihood. Because we state IMMA in terms of all values of $m, x_{\mathrm{obs}}$, and $w$, then this result will hold both in our realized sample and in repeated samples. Thus, both Bayesian inference and general frequentist inference are justified under this result (Seaman et al. 2013). From here on, we thus refer to $L(\theta, \gamma)$ as the observed-data likelihood, where its dependence on IMMA is implicit.

\section{An EM Algorithm for Missing Data and Measurement Error}

The EM algorithm is commonly used to maximize likelihoods intractable due to missingness. In this section, we show how we can incorporate measurement error into the standard EM algorithm for missing data. For expository purposes, we assume that the true values of the measurement error distribution parameters, $\gamma$, are known and, thus, fixed. In the Heteroskedastic Measurement Error subsection, we consider extensions for when $\gamma$ is estimated.

The object in the EM algorithm is the expectation of the log likelihood of the complete data, averaged across the missing data given a current guess of the parameters:

$$
Q\left(\theta \mid \theta^{(t)}\right)=\int \log \left[p\left(x_{\mathrm{mis}}, x_{\mathrm{obs}}, w \mid \theta, \gamma\right)\right] p\left(x_{\mathrm{mis}} \mid x_{\mathrm{obs}}, w, \theta^{(t)}, \gamma\right) \mathrm{d} x_{\mathrm{mis}}
$$

Note that the distribution of the missing data which we are averaging across fixes the value of $\theta$, while the value in the complete-data log likelihood is allowed to vary. The EM algorithm proceeds in two steps. First, in the E-step, we compute the function $Q\left(\theta \mid \theta^{(t)}\right)$, which involves calculating the posterior distribution of the missing data, conditional on the observed. 
The second step, called the M-step, maximizes $Q\left(\theta \mid \theta^{(t)}\right)$ with respect to $\theta$. This becomes the new candidate value, $\theta^{(t+1)}$, and the algorithm iterates until convergence. Under suitable conditions ( $\mathrm{Wu} 1983$ ), the EM algorithm converges to the MLE of $\theta$ under the observed-data likelihood, $L(\theta, \gamma)$.

The complete-data log likelihood is straightforward in our general setup:

$$
\log \left[p\left(x_{\mathrm{mis}}, x_{\mathrm{obs}}, w \mid \theta, \gamma\right)\right]=\log \left[p\left(x_{\mathrm{mis}}, x_{\mathrm{obs}} \mid \theta\right)\right]+\log \left[p\left(w \mid x_{\mathrm{mis}}, x_{\mathrm{obs}}, \gamma\right)\right] \text {. }
$$

Because the last term only depends on the known (mis)measurement process, it will not factor into the maximization of $Q\left(\theta \mid \theta^{(t)}\right)$. This follows from our (implicit) assumption that the parameters of the measurement process are distinct from the parameters of the target data $x$. The measurement information will enter the EM algorithm through its influence on the posterior distribution of $x_{\text {mis: }}$ :

$$
p\left(x_{\mathrm{mis}} \mid x_{\mathrm{obs}}, w, \theta^{(t)}, \gamma\right) \propto p\left(x_{\mathrm{mis}} \mid x_{\mathrm{obs}}, \theta^{(t)}\right) p\left(w \mid x_{\mathrm{mis}}, x_{\mathrm{obs}}, \gamma\right) .
$$

Thus, we can write the objective function in this case as

$$
Q\left(\theta \mid \theta^{(t)}\right)=\int \log \left[p\left(x_{\mathrm{mis}}, x_{\mathrm{obs}} \mid \theta\right)\right] p\left(x_{\mathrm{mis}} \mid x_{\mathrm{obs}}, \theta^{(t)}\right) p\left(w \mid x_{\mathrm{mis}}, x_{\mathrm{obs}}, \gamma\right) \mathrm{d} x_{\mathrm{mis}} .
$$

Because equation (19) is a posterior for the missing data and equation (18) is a complete-data log likelihood, all of the properties of the general EM algorithm will apply to it here with the form given by equation (20). Namely, this algorithm will converge to the MLE of $\theta$ under the observed-data likelihood. If we replace the true parameters, $\gamma$, with a consistent estimate, $\hat{\gamma}$, the EM algorithm will still converge to a consistent estimator of the completedata parameters by standard asymptotic arguments (Newey and McFadden 1994). While estimating $\gamma$ does not affect the consistency of our EM estimator, it does affect the variance of the asymptotic distribution. In order to achieve consistent variance estimates, we can rely on the nonparametric bootstrap.

\section{MO}

Analyzing the true values of the data $x$ would be much easier than the observed data since the mismeasured and missing components contribute to the likelihood (equation (8)) in complicated ways. Thus, MO seeks to form a series of complete, ideal data sets: $x^{(1)}, x^{(2)}, \ldots, x^{(B)}$. Each of these overimputed data sets is of the form $x^{(b)}=\left(x_{\mathrm{obs}}, x_{\text {mis }}^{(b)}\right)$, so that the perfectly 
measured data are constant across the overimputations. We refer to this as overimputation because we replace observed data $w$ with draws from an imputation model for $x_{\text {mis }}$. To form these overimputations, we take draws from the posterior predictive distribution of the unobserved data:

$$
\left(x_{\text {mis }}^{(b)}\right) \sim p\left(x_{\text {mis }}^{(b)} \mid x_{\mathrm{obs}}, w\right)=\int p\left(x_{\text {mis }}^{(b)} \mid x_{\mathrm{obs}}, w, \theta, \gamma\right) p\left(\theta, \gamma \mid x_{\mathrm{obs}}, w\right) d \theta d \gamma
$$

Once we have these $B$ overimputations, we can simply run $B$ separate analyses on each data set and combine them using straightforward rules in one of the two standard ways in MI. See The Foundation: A Multiple Imputation Model subsection in BHK.

Equation (21) suggests one way to create MIs: (1) draw $\theta^{(b)}$ from its posterior $p\left(\theta \mid x_{\mathrm{obs}}, w, \gamma\right)$ and then (2) draw $\left(x_{\text {mis }}^{(b)}\right)$ from $p\left(x_{\text {mis }}^{(b)} \mid x_{\mathrm{obs}}, w, \theta^{(b)}, \gamma\right)$. Usually, these procedures are implemented with either data augmentation (i.e., Gibbs sampling) or the EM algorithm combined with an additional sampling step. We focus here on how our method works in the EM algorithm, since these two approaches are closely linked and often lead to similar inferences (Honaker and King 2010; King et al. 2001; Schafer 1997). The EM algorithm from An EM Algorithm for Missing Data and Measurement Error subsection will give us either an MLE or maximum a posteriori (MAP) estimate of the parameters of the complete data, $\hat{\theta}$. With this estimate, there are various ways to draw from the predictive posterior distribution. Here, we apply the EM algorithm to a series of data sets drawn using the nonparametric bootstrap and use the estimated value of $\hat{\theta}_{b}$ in that bootstrapped sample as a draw from the posterior of $\theta$. Of course, $\mathrm{MO}$ is not limited to the EM algorithm; we could replace it with a suitable Markov chain Monte Carlo (MCMC) approach as well.

\section{A Specific Model Within the Class}

In the above description of the model, we have left the distributions unspecified. To implement a specific model, we must of course specify this additional information. To make our estimation approach practical, we narrow the class of allowable data generation processes to any distribution that possesses the property of statistical duality. This is a simple property (related to self-conjugacy in Bayesian analysis) possessed by a variety of distributions, such as normal, Laplace, gamma, inverse gamma, Pareto, and others (Bityukov et al. 2006). ${ }^{1}$ Here, we offer the most common choice in practice, which is that the complete (but partially unobserved) data $(x)$ are multivariate normal with mean $\mu$ and covariance 
$\Sigma$. This implies that any conditional distribution of the ideal is also normal.

The abovementioned measurement error distribution is, in its most general form, a function of the entire ideal data vector, $x$, and some parameters, $\gamma$. As noted by Stefanski (2000), all approaches to correcting measurement error must include additional information about this distribution. In the simple but powerful special case, we assume that $w_{i j} \stackrel{\text { ind }}{\sim} \mathcal{N}\left(x_{i j}, \lambda_{i j}^{2}\right)$ where the measurement error variance $\lambda_{i j}^{2}$ is known or estimable using techniques from the third section. Our assumption corresponds to that of classical measurement error, yet our modified EM algorithm can handle more general cases than this. If the measurement error is known to be biased or dependent upon another variable, we can simply adjust the cell-level means above and proceed as usual. Essentially, one must have knowledge of how the variable was mismeasured. The simulation results in the Robustness to Violating Assumptions subsection further indicate that $\mathrm{MO}$ is robust to these assumptions in certain situations.

With the aforementioned measurement error model, the normality of the data makes the calculation of the sufficient statistics straightforward. In a slight abuse of notation, we can gather the independent measurement error distributions on $w_{i j}$ into a multivariate normal with mean $x_{\text {mis }, i}$ and covariance matrix $\Lambda_{i}=\lambda_{i}^{2} I$, where $\lambda_{i}^{2}=\left\{\lambda_{i j}^{2} ; m_{i j}=1\right\}$ and $I$ is the identity matrix with dimension equal to $\sum_{j} \mathbb{I}\left\{m_{i j}=1\right\}$. To ease exposition, let $\theta=(\mu, \Sigma)$ and consider the case without fully missing values. When $x$ comes from an exponential family, the E-step involves calculating the expected value of the complete-data sufficient statistics, marginalized across the missing data:

$$
\begin{aligned}
E\left[T(x) \mid x_{\mathrm{obs}}, w, \theta^{(t)}\right] & =\int T(x) p\left(x_{\mathrm{mis}} \mid x_{\mathrm{obs}}, \theta^{(t)}\right) p\left(w \mid x_{\mathrm{mis}}, \gamma\right) \mathrm{d} x_{\mathrm{mis}}, \\
& =\int T(x) \prod_{i} p\left(x_{i, \mathrm{mis}} \mid x_{i, \mathrm{obs}}, \theta^{(t)}\right) p\left(w_{i} \mid x_{i, \mathrm{mis}}, \Lambda_{i}\right) \mathrm{d} x_{i, \mathrm{mis}} .
\end{aligned}
$$

Here, $T(x)$ is the set of sufficient statistics for the multivariate normal. Calculating this expression is equivalent to evaluating $Q\left(\theta \mid \theta^{(t)}\right)$ in equation (20).

In order to calculate the expectation in equation (23), we must know the full conditional distribution, which is $p\left(x_{i, \text { mis }} \mid x_{i, \mathrm{obs}}, w_{i}, \theta, \Lambda_{i}\right) \propto p\left(x_{i, \mathrm{mis}} \mid\right.$ $\left.x_{i, \text { obs }}, \theta\right) p\left(w_{i} \mid x_{i, \text { mis }}, \Lambda_{i}\right)$. Note that each of the distributions is (possibly multivariate $)$ normal, with $\left[x_{i, \text { mis }} \mid x_{i, \mathrm{obs}}, \theta\right] \sim \mathcal{N}\left(\mu_{\mathrm{e} \mid \mathrm{o}}, \Sigma_{\mathrm{e} \mid \mathrm{o}}\right)$ and $\left[w_{i} \mid x_{i, \text { mis }}, \Lambda_{i}\right] \sim$ $\mathcal{N}\left(x_{i, \text { mis }}, \Lambda_{i}\right)$, where $\left(\mu_{\mathrm{e} \mid \mathrm{o}}, \Sigma_{\mathrm{e} \mid \mathrm{o}}\right)$ are deterministic functions of $\theta$ and $x_{i, \text { obs. }}$ This distribution amounts to the regression of $x_{i, \text { mis }}$ on $x_{i, \mathrm{obs}}$. If the values were simply missing, rather than measured with error, then the E-step would 
simply take the expectations with respect to this conditional expectation. With measurement error, we must combine these two sources of information. Using standard results on the normal distribution, we write the full conditional as

$$
\begin{aligned}
\left(x_{i, m i s} \mid x_{i, o b s}, w_{i}, \theta^{(t)}, \Lambda_{i}\right) \sim \mathcal{N}\left(\mu^{*}, \Sigma^{*}\right), & \Sigma^{*}=\left(\Lambda_{i}^{-1}+\Sigma_{\mathrm{e} \mid \mathrm{o}}^{-1}\right)^{-1}, \\
\mu^{*} & =\Sigma^{*}\left(\Lambda_{i}^{-1} w_{i}+\Sigma_{\mathrm{e} \mid \mathrm{o}}^{-1} \mu_{\mathrm{e} \mid \mathrm{o}}\right) .
\end{aligned}
$$

We simply change our E-step to calculate this expectation for each cell measured with error and proceed with the M-step as usual. ${ }^{2}$ That is, we would find the values of $\mu^{(t+1)}$ and $\Sigma^{(t+1)}$ that maximize the likelihood with these updated sufficient statistics in equation (23). Note that while we assume that the measurement errors on different variables are independent, one could incorporate dependence into $\Lambda_{i}$. The result in equation (24) is identical to the results in Honaker and King (2010), when we set a prior distribution for $x_{i \text {,mis }}$ that is normal with mean $w_{i}$ and variance $\Lambda_{i}$. See their article for additional implementation details.

Finally, we note that the imputation of purely missing values for a given observation will not directly depend on imputations of the mismeasured value for that observation. This is because imputations are always computed from a regression of a missing value on the observed data for that observation. Thus, since both missing and mismeasured variables are missing for a given observation, the imputation of one will not affect the other. If there is gold standard data for a mismeasured value, then the mismeasured variable is observed and, thus, the imputations of the missing values can depend on that variable. In this case, the mismeasurement model can influence the imputed values for the missing data through its influence on the completedata parameters. This is appropriate, though, because the mismeasurement model helps to push the complete-data parameters to reflect the true, underlying data, which is exactly the information being used to impute missing values with gold standard measurements in the mismeasured variable.

\section{Directly Estimating Measurement Error Variances}

Earlier we assumed that the amount of measurement error is known or estimable from the data. In the Specifying or Estimating the Measurement Error Variance section of BHK, we show how to analyze variables with a known proportion of measurement error or how to bound estimates when it is unknown. However, auxiliary variables often exist, which can provide 
feasible estimates of the level of measurement error. ${ }^{3}$ The other models in the literature reviewed in MO subsection all rely on the existence of such auxiliary information. First, when replicated correlated proxies are available, we show how to estimate $\lambda_{j}^{2}$ for variable $\mathrm{j}$ directly (Multiple Proxies subsection). Second, we show how to proceed when $\lambda_{i j}^{2}$ varies over the observations $i$ or when gold standard observations are available (Heteroskedastic Measurement Error subsection).

\section{Multiple Proxies}

When multiple proxies (or "repeated measures") of the same true variable are available, we can use relationships among them to provide point estimates of the required variances, and to set the priors in MO. For example, suppose for the same true variable $x_{i j}$ we have two unbiased proxies with normal errors that are independent after conditioning on $x_{i j}$ :

$$
w_{i 1}=x_{i j}+u_{i}: u_{i} \sim \mathcal{N}\left(0, \lambda_{j}^{2}\right), \quad w_{i 2}=a x_{i j}+b+v_{i}: v_{i} \sim \mathcal{N}\left(0,\left(c \lambda_{j}\right)^{2}\right),
$$

where $a, b, c$ are unknown parameters that rescale the additional proxy measure to a different range, mean, and different degree of measurement error. The covariances and correlations between these proxies can be solved as $E\left[\operatorname{cov}\left(w_{1}, w_{2}\right)\right]=a \operatorname{var}\left(x_{j}\right)$ and $E\left[\operatorname{cor}\left(w_{1}, w_{2}\right)\right]=\gamma \operatorname{var}\left(x_{j}\right) / \operatorname{var}\left(w_{1}\right)$, where $a$ is one of the abovementioned scale parameters and $\gamma$ is a ratio:

$$
\gamma^{2}=a^{2} \frac{\operatorname{var}\left(w_{1}\right)}{\operatorname{var}\left(w_{2}\right)}=\frac{\operatorname{var}\left(x_{j}\right)+\operatorname{var}(u)}{\operatorname{var}\left(x_{j}\right)+\left(c^{2} / a^{2}\right) \operatorname{var}(u)},
$$

where all of these expectations are over units. If the measurement error is uncorrelated with $x_{j}$, the variances decompose as $\lambda_{j}^{2}=\operatorname{var}\left(w_{1}\right)-\operatorname{var}\left(x_{j}\right)$. This leads to two feasible estimates of the error variances for setting priors. First,

$$
s^{2}(u)=\operatorname{var}\left(w_{1}\right)-\operatorname{cov}\left(w_{1}, w_{2}\right)=\operatorname{var}\left(w_{1}\right)-\operatorname{var}\left(x_{j}\right) a,
$$

which is exactly correct when $a=1$, that is, when $w_{2}$ is on the same scale (with possibly differing intercept) as $w_{1}$. Similarly,

$$
s^{2}(u)=\operatorname{var}\left(w_{1}\right)\left(1-\operatorname{cor}\left(w_{1}, w_{2}\right)\right)=\operatorname{var}\left(w_{1}\right)-\operatorname{var}\left(x_{j}\right) \gamma,
$$

which is exactly correct when $c=a \Leftrightarrow \gamma=1$, that is, the second proxy has the same relative proportion of error as the original proxy. 


\section{Heteroskedastic Measurement Error}

In some applications, the amount of measurement error may vary across observations. Although most corrections in the literature ignore this possibility, it is easy to include in the MO framework, and doing so often makes estimation easier. To include this information, we add a subscript $i$ to the variance of the measurement error: $p\left(w_{i} \mid x_{i j}, \lambda_{i j}^{2}\right)$. We then consider two examples.

First, suppose the data include some observations measured with error and some without error. For the gold standard observations, we have $w_{i j}=x_{i j}$, or equivalently $\lambda_{i j}^{2}=0$. The imputation model would only overimpute cell values measured with error, leaving the "gold standard" observations as is. If the other observations have a common error variance, $\lambda_{j}^{2}$, then we can easily estimate this quantity, since the variance of the gold standard observations is $\sigma_{x}^{2}$ and the mismeasured observations have variance $\sigma_{x}^{2}+\lambda_{j}^{2}$. This leads to the feasible estimator

$$
\hat{\lambda}_{j}^{2}=\hat{\sigma}_{m m}^{2}-\hat{\sigma}_{g s}^{2},
$$

where $\hat{\sigma}_{m m}^{2}$ is the estimated variance of the mismeasured observations and $\hat{\sigma}_{g s}^{2}$ is the estimated variance of the gold standard observations.

As a second special case of Heteroskedastic measurement error, $\mathrm{MO}$ can handle situations where the variance is a linear function of another variable. That is, when $\lambda_{i j}^{2}=r z_{i}$, where $z_{i}$ is variable and $r$ is the proportional constant relating the variable to the error variance. If we know $r$ (or we can estimate it through variance function approaches), then we can easily incorporate this into the prior above using $w_{i} \sim \mathcal{N}\left(w_{i} \mid x_{i j}, r Z_{i}\right)$.

\section{Robustness to Categorical Variables Measured With Error}

While our imputation model assumes the data are drawn from a multivariate normal distribution, non-normal variables, such as categorical variables, can be included in the imputation and can even be overimputed for measurement error. It is well known in the MI literature that imputation via a normal model works well for categorical variables, and indeed as well as models designed especially for categorical variables and even when the analysis model is nonlinear (Schafer 1997; Schafer and Olsen 1998).

To illustrate how these findings extend to the MO context, we construct a simulation study with measurement error on a binary variable. To do so, we use a similar setup to BHK, except that both the underlying latent variable (which we call $x_{i}$ ) and the mismeasured proxy $\left(w_{i}\right)$ are each a five-category 
ordinal variable. That is, $x_{i}$ is drawn from a binomial distribution with five trials and a 0.2 probability of success. Note that this forces $x_{i}$ to exhibit skew. To create measurement error, we add $u_{i} \sim \mathcal{N}(0,0.2)$ to the categorical variable and then use cutoffs at $0.5,1.5,2.5,3.5$, and 4.5 to force the noisy variable to also be categorical. This leads to roughly 20 percent of the observations to be miscoded and leads to a proportion of variance due to measurement error, $\rho$, of 0.2 . The goal, as before, is to correctly recover the effect of $x_{i}$ on $y_{i}$, where we have $y_{i}=\beta_{0}+\beta_{1} x_{i}+\beta_{2} z_{i}+\varepsilon_{i}{ }^{4}$

We follow the same procedure as in the Specifying or Estimating the Measurement Error Variance section of BHK and run MO with a range of assumptions about $\rho$, where $\rho$ is the proportion of variance due to measurement error. In this simulation, we use the MO in its default form and leave the values overimputed from the normal model as is, without rounding them to their nearest category. Figure 1 shows the results of these simulations, with a vertical dashed line that represents the amount of variance due to the continuous measurement error, $u_{i}$. Note that when we discretize the mismeasured variable, it reduces the variance slightly; the true $\rho$ will be slightly less than the one denoted on the plot. The results from this more challenging test are largely similar to our earlier simulations. Even in the face of skewed, categorical latent and observed variables, $\mathrm{MO}$ is able to recover good estimates of the slope, $\beta_{1}$, around the true amount of measurement error. Further, MO minimizes the root mean square error at the point as well. While this is a promising approach for categorical variables, tailored methods for misclassification, such as Katz and Katz (2010), may outperform MO in applications that primarily focus on measurement errors on binary variables.

\section{Robustness to Correlated Measurement Errors}

In this section and the next, we show how MO is robust to data problems that may occur in a large number of settings and applications. We show here how $\mathrm{MO}$ is robust to theoretical measurement dilemmas that occur regularly in social science data.

Until now, we have assumed that measurement error is independent of all other variables. We now show how to relax this assumption. Many common techniques for treating measurement error make this strong assumption and are not robust when it is violated. For example, probably the most commonly implemented measurement error model (in the rare cases that a correction is attempted at all) is the classic errors-in-variables (EIV) model. We thus first briefly describe the EIV model to illustrate the strong assumptions required. The EIV model is also a natural point of comparison to MO, since both can 


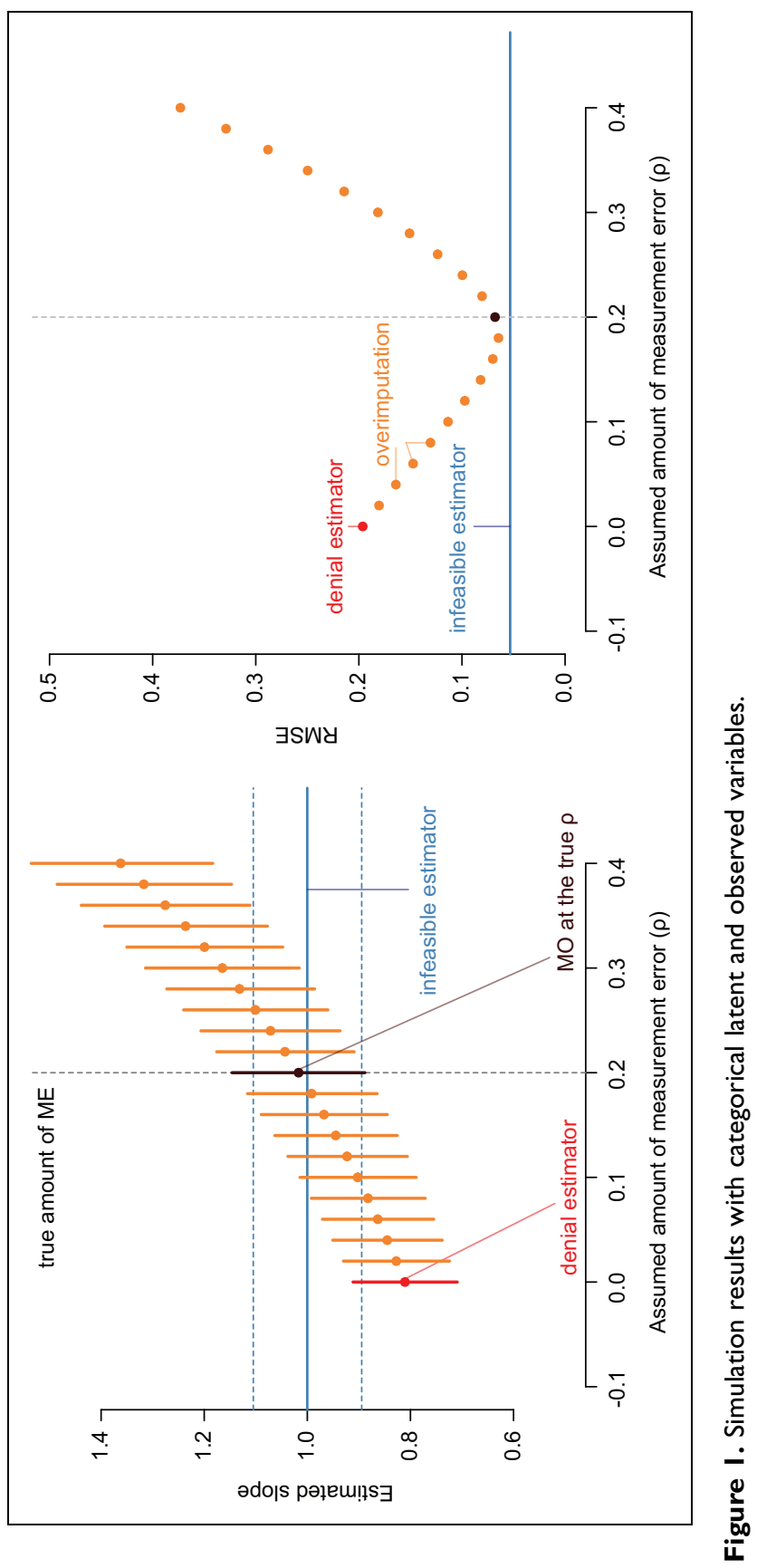


be thought of as replacing mismeasured observations with predictions from auxiliary models.

\section{The Foundation: The EIV Model}

As mentioned earlier, assume $y_{i}$ and $x_{i}$ are jointly normal with parameters as in BHK, equation (2). Suppose instead of $x_{i}$ we have a set of proxy variables which are measures of $x_{i}$ with some additional normally distributed random noise:

$$
\begin{aligned}
w_{i 1}=x_{i}+u_{i}, & u_{i} \sim \mathcal{N}\left(0, \sigma_{u}^{2}\right), \\
w_{i 2}=x_{i}+v_{i}, & v_{i} \sim \mathcal{N}\left(0, \sigma_{v}^{2}\right),
\end{aligned}
$$

ordered such that $\sigma_{u}^{2}<\sigma_{v}^{2}$, making $w_{1}$ the superior of the two proxies as it has less noise.

Suppose the true relationship is $y_{i}=\alpha x_{i}+\varepsilon_{i 1}$, and we instead use the best available proxy and estimate $y_{i}=\beta w_{i 1}+\varepsilon_{i 2}=\beta\left(x_{i}\right)+\beta\left(u_{i}\right)+\varepsilon_{i 2}$. We then get some degree of attenuation $0<\beta<\alpha$ since the coefficient on $u_{i}$ should be zero. This attenuation is shown in one example in the right-hand side of Figure 2 where the relationship between $y_{i}$ and $w_{1}$ shown in red is weaker than the true relationship with $x_{i}$ estimated in the left graph and copied in black on the right.

In this simple example, we can calculate the expectation of this attenuation. The coefficient on $w_{i 1}$ will be

$$
E\left[\hat{\beta}_{1}\right]=E\left[\frac{\sum_{i}\left(x_{i}+u_{i}-(\overline{x+u})\right)\left(y_{i}-\bar{y}\right)}{\sum_{i}\left(x_{i}+u_{i}-(\overline{x+u})\right)^{2}}\right]=\frac{\sum_{i}\left(x_{i}-\bar{x}\right)\left(y_{i}-\bar{y}\right)}{\sum_{i}\left(x_{i}-\bar{x}\right)^{2}+\sigma_{u}^{2}},
$$

where $\overline{x+u}$ and $\bar{x}$ are the sample means of $w_{i 1}$ and $x_{i}$, respectively. The last term in the denominator, $\sigma_{u}^{2}$, causes this attenuation. If the variance of the measurement error is zero, the term drops out and we get the correct estimate. As the measurement error increases, the ratio tends to zero.

The coefficients in the EIV approach can be estimated either directly or in two stages. A two-stage estimation procedure is the common framework to build intuition about the model and the role of the additional proxy measure. In this approach, we first obtain estimates of $x_{i}$ from the relationship between the $w$ 's since they only share $x_{i}$ in common, $\hat{w}_{i 1}=\hat{\gamma} w_{i 2}$, and then use these predictions to estimate $y_{i}=\delta \hat{w}_{i 1}+\varepsilon_{i 3}$, where now $\hat{\delta}$ is an unbiased estimate of $\alpha$. The relationship between the two proxy variables 


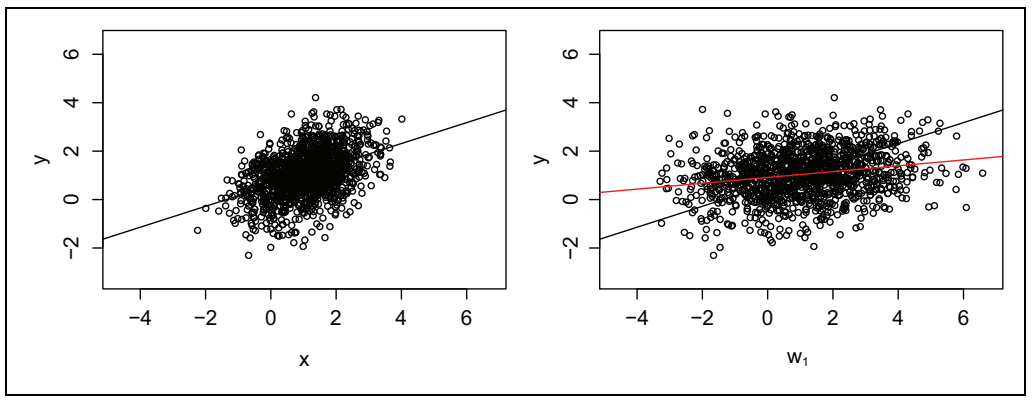

Figure 2. On the left, we see the true relationship between $y_{i}$ and the latent $x_{i}$. When the mismeasured proxy $w_{i 1}$ is used instead, the estimated relationship (shown in red) is attenuated compared to the true relationship (shown in black in both graphs).

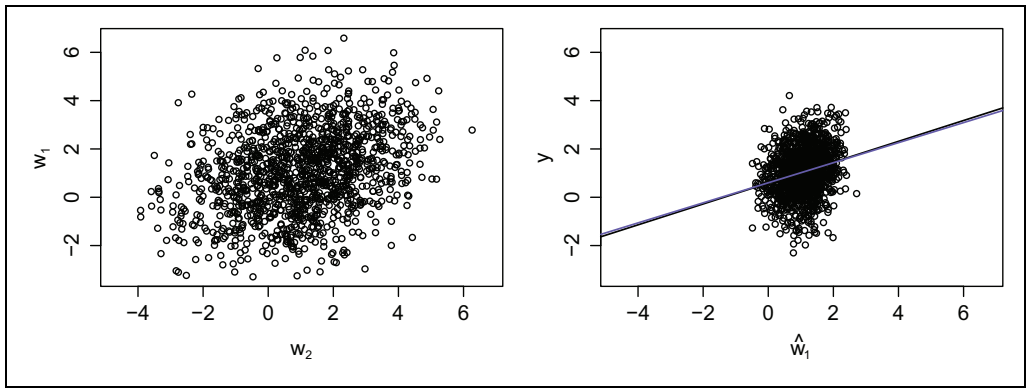

Figure 3. The relationship between two mismeasured proxy variables (left) and the relationship between the predicted values from this model and $y$ (right). The relationship here, shown in green, recovers the true relationship, shown in black.

is shown in the left-hand side of Figure 3, and the relationship between the first stage predicted values of $w_{i 1}$ and $y_{i}$ is shown in blue in the right figure. This coincides almost exactly with the true relationship still shown in black in this figure.

In Figure 4, we illustrate how the EIV model performs in data that meet its assumptions. The black distributions represent the distribution of coefficients estimated when the latent data $x^{*}$ are available in a simulated data set of size $500 .^{5}$ The naive regressions that do not account for measurement error are shown in red in both graphs. The coefficient on $w_{i 1}$ is attenuated toward zero (bottom panel). The estimated constant term is biased upward to compensate (top panel). In each simulated data set, 


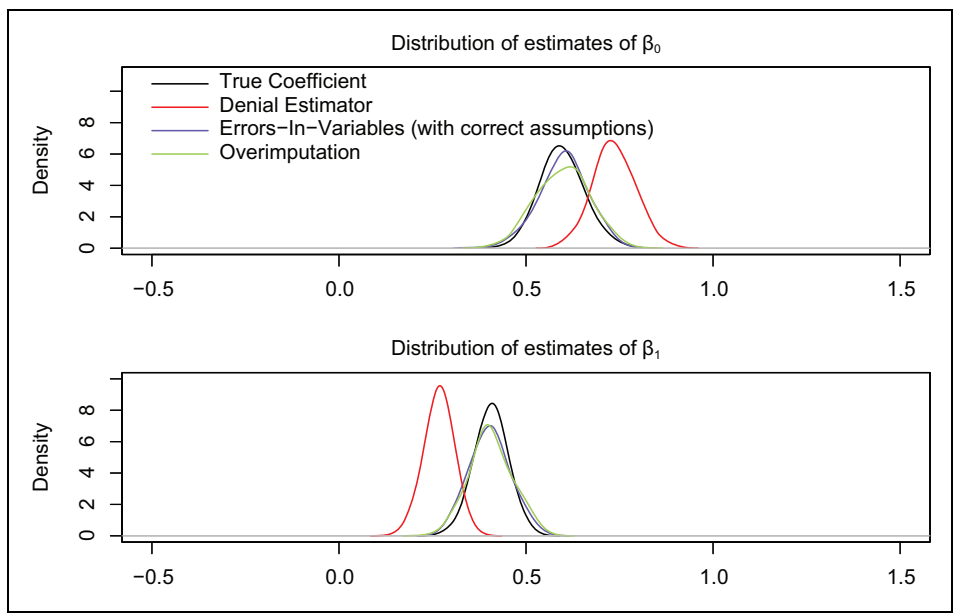

Figure 4. Coefficients estimated from variables with measurement error (shown in red) attenuate the effect of the independent variable toward zero and also bias the constant in compensation. The estimates recovered from the errors-in-variables (EIV) model (in green) recover the true distribution, but are of course less efficient (slightly higher variance) than the original latent data (in black).

we use the EIV model (in blue) and see that the distribution of estimated parameters using the proxies resembles the distribution using the latent data, although with slightly greater variance. Thus, there is some small efficiency loss, but the EIV model clearly recovers unbiased estimates when its assumptions are met.

We also run the MO on the same simulated data sets in which we ran the EIV model. The distribution of coefficients (which we present subsequently) recovers the distribution that would have been estimated if the latent data had been available. Thus, in the simple setting where the assumptions of the EIV model are met, our approach performs equivalently.

\section{Robustness to Violating Assumptions}

If we think of the coefficient on $x_{i}$ as the ratio of $\operatorname{cov}(x, y)$ to $\operatorname{var}(x)$, then the attenuation in equation (32) is being driven by the fact that $\operatorname{var}\left(w_{1}\right)>\operatorname{var}(x)$ because of the added measurement error. Therefore, $\operatorname{var}\left(w_{1}\right)$ is not a good estimate of $\operatorname{var}(x)$, even though $\operatorname{cov}\left(w_{1}, y\right)$ is a good measure of $\operatorname{cov}(x, y)$. With this in mind, the numerically simpler - but equivalent — one stage approach to the EIV model has a useful intuition. We substitute $\operatorname{cov}\left(w_{1}, w_{2}\right)$ as an estimate 
of $\operatorname{var}(x)$ because $w_{1}, w_{2}$ only covary through $x$. Thus, we have as our estimate of the relationship ${ }^{6}$ :

$\hat{\delta}=\frac{\sum_{i}\left(w_{i 1}-\bar{w}_{1}\right)\left(y_{i}-\bar{y}\right)}{\sum_{i}\left(w_{i 1}-\bar{w}_{1}\right)\left(w_{i 2}-\bar{w}_{2}\right)}=\frac{\sum_{i}\left(x_{i}-\bar{x}\right)\left(y_{i}-\bar{y}\right)+u_{i}\left(y_{i}-\bar{y}\right)}{\sum_{i}\left(x_{i}-\bar{x}\right)^{2}+u_{i}\left(x_{i}-\bar{x}\right)+v_{i}\left(x_{i}-\bar{x}\right)+u_{i} v_{i}}$.

In order to recover the true relationship between $x$ and $y$, we need the last term in the numerator and the last three in the denominator to drop out of equation (33). To obtain a consistent estimate, then EIV requires: (1) $E\left(u_{i} \cdot y_{i}\right)=0$, (2) $E\left(u_{i} \cdot x_{i}\right)=0$ and $E\left(v_{i} \cdot x_{i}\right)=0$, and (3) $E\left(u_{i} \cdot v_{i}\right)=0$. Indeed, when these conditions are not met, the resulting bias in the EIV correction can easily be larger than the original bias caused by measurement error. However, as we now show in the following three subsections, MO is robust to violations of all but the last condition.

Measurement error correlated with $y$. The first of the conditions for EIV to work is that the measurement error is unrelated to the observed dependent variable. As an example of this problem, we might think that infant mortality is related to international aid because donors want to reduce child deaths. If countries receiving aid are intentionally underreporting infant mortality, to try to convince donors the aid is working, then the measurement error in infant mortality is negatively correlated with the dependent variable, foreign aid. If instead countries searching for aid are intentionally overreporting infant mortality as a stimulus for receiving aid, then measurement error is positively correlated with the dependent variable. Both scenarios are conceivable. This problem with the EIV approach is well known, because the EIV model has an instrumental variables framework, and this is equivalent to the problem of assuring the instrument is exogenous of $y$ in the more common usage of instrumental variables as a treatment for endogeneity.

In Figure 5(a), we demonstrate this bias with simulated data. ${ }^{7}$ The violet densities show the distribution of parameter estimates when there is negative correlation of .1 (dashed) and .3 (solid) between the measurement error and the dependent variable. In the latter case, the bias in the correction has exceeded the original bias from measurement error, still depicted in red. The blue densities show that positive correlation of the errors creates bias of similar magnitude in the opposite direction. Again, the size of the bias can be greater than that originally produced by the measurement error we were attempting to correct. Moreover, the common belief with measurement 


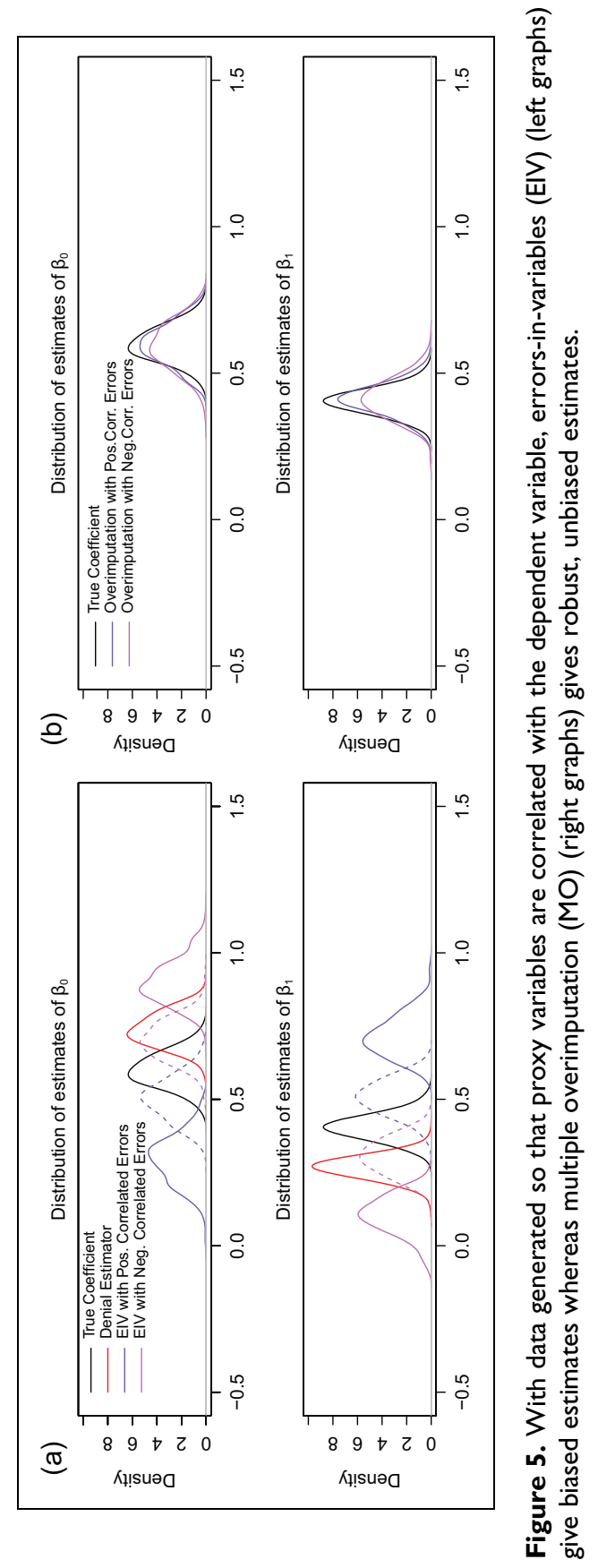


issues is that any resulting bias attenuates the coefficients so that estimates are at least conservative; however, here we see that the bias in the EIV approach can actually exaggerate the magnitude of the effect.

We now analyze the same simulated data sets with MO. To apply the MO model, we estimate the measurement error variance from the correlation between the two proxies and leave the mean set to the better proxy. As Figure 5(b) indicates, $\mathrm{MO}$ recovers the distribution of coefficients for each of the data generation processes: The violet line represents the distribution when there is positive correlation while the blue line (barely visible under the other two) represents the distribution with negative correlation. All three distributions are close to each other and close to the true distribution in black using the latent data.

Measurement error correlated with the latent true value. The second requirement of the EIV model is that the measurement error is independent of the latent variable. If, for example, we believe that income is poorly measured, and wealthier respondents feel pressure to underreport their income while poorer respondents feel pressure to overreport, then the measurement error can be correlated with the latent variable.

In Figure 6(a), we demonstrate the bias this produces in EIV. Here, the error in $w_{i 2}$ is correlated with the latent $x_{i}{ }^{8}$ The biases are in the opposite directions as when the correlation is with $y_{i}$, although lesser in magnitude. Errors positively correlated with $x_{i}$ lead to attenuated coefficients, and negatively correlated errors lead to overstated coefficients, as shown by the blue and violet distributions in Figure 6(a), respectively. Dashed lines are the result of small levels of correlations $( \pm 0.1)$ and the solid lines a greater degree $( \pm 0.3)$.

The coefficients resulting from MO, with measurement error variance estimated from the correlation between the proxies, are contrasted in Figure 6(b). In the positive correlation case, the distributions recover the same parameters. Because they sit on top of each other, only the simulations with the greatest correlation $( \pm 0.3)$ are shown. For both parameters, with positive correlation, the MO estimates reveal no bias. With negative correlation, neither equation (27) nor (28) gives good estimates of the measurement error. Here, we see bias in the coefficients similar to the EIV model. The key cause of the bias is the inability to judge the measurement error when the proxy is negatively correlated with the true latent $x$. If we had additional auxiliary information to set the prior variance for $w_{i 1}$ correctly, and used $w_{i 2}$ simply as a predictor, we would recover unbiased estimates with the MO model, as is shown in the simulations in Figure 6(c). This follows the results seen in equation (1) and in Figure 3 of BHK. 


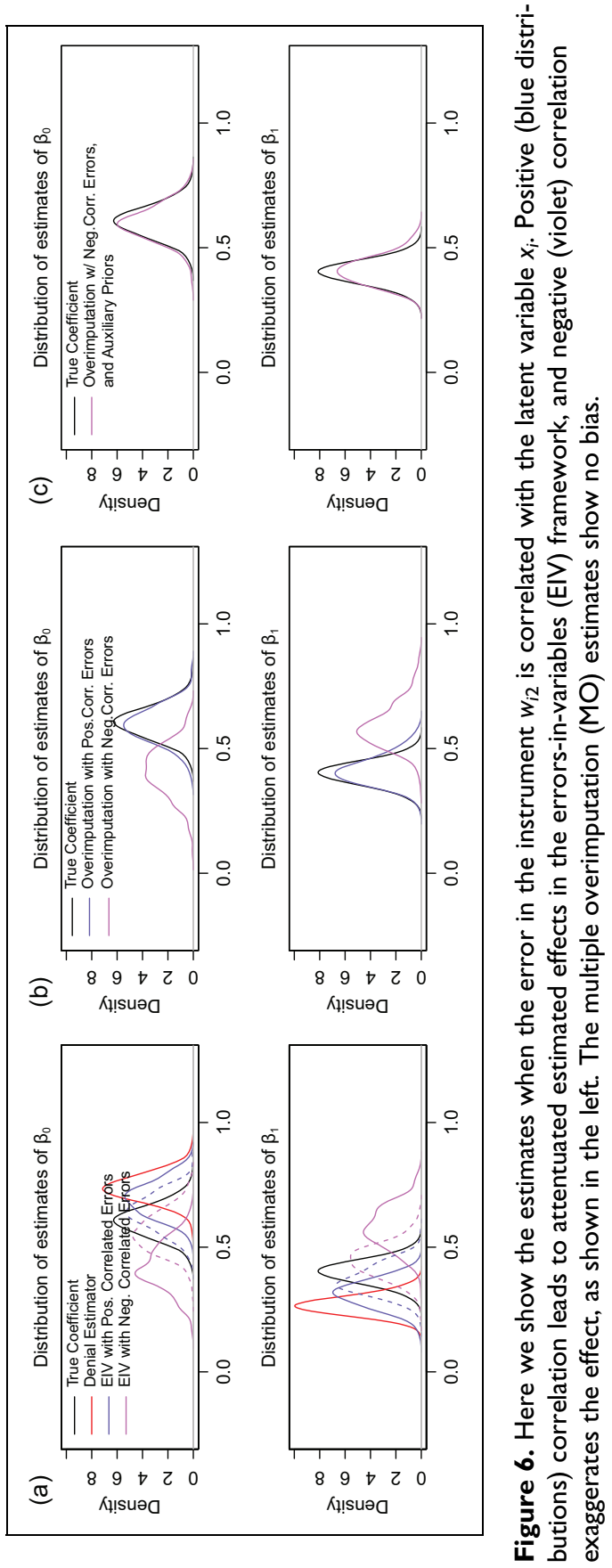



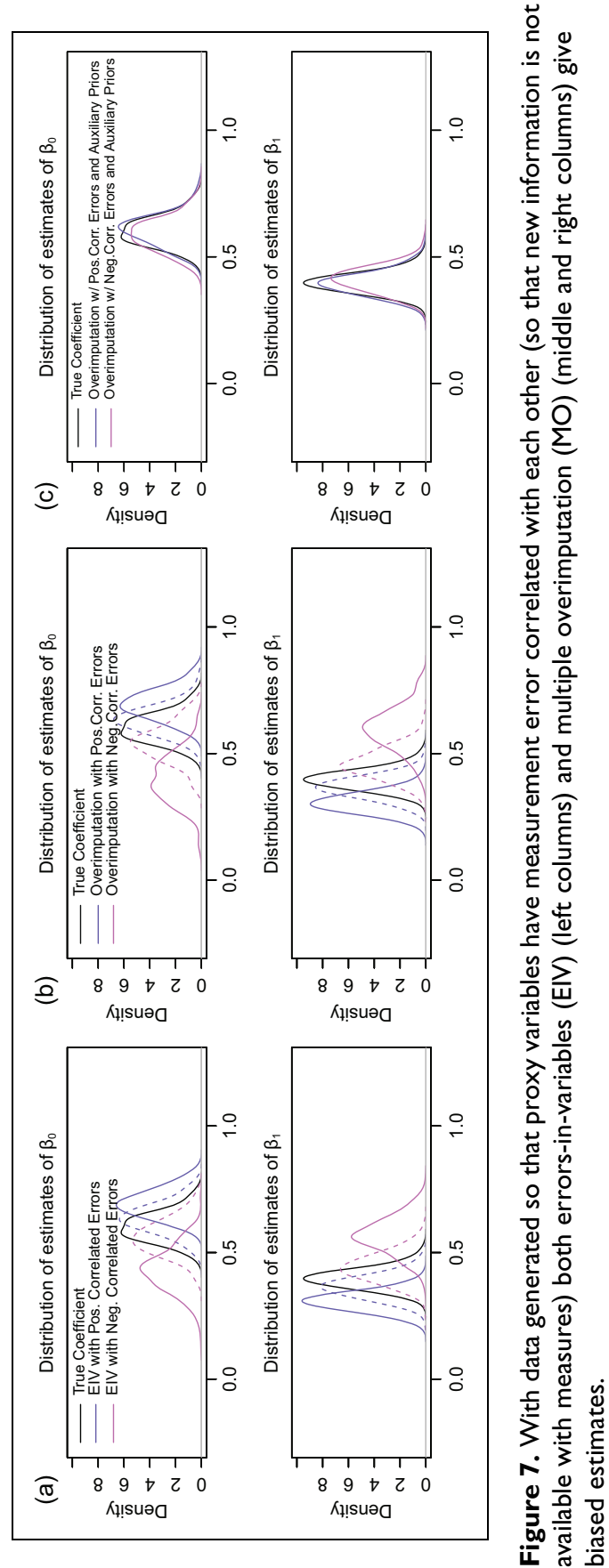
Measurement errors that covary across proxies. The final condition requires the errors in the proxies be uncorrelated. If all the alternate measures of the latent variable have the same error process, then the additional measures provide no additional information. For example, if we believe gross domestic product (GDP) is poorly measured, it is not enough to find two alternate measures of GDP; we also need to know that those sources are not making the same errors in their assumptions, propagating the same errors from the same raw sources, or contaminating each other's measure by each making sure their estimates are in line with other published estimates. To the extent the errors in the alternate measures are correlated, then $\sigma_{u v}$ will attenuate the estimate in the same manner as $\sigma_{u}^{2}$ did originally.

Thus, we now simulate data where the measurement errors across alternate proxies are correlated. ${ }^{9}$ Figure 7(a) shows positively (negatively) correlated errors lead to bias in the EIV estimates that are in the same (opposite) direction as the original measurement error. Intuitively, if the errors are perfectly correlated, both the original proxy and the alternate proxy would be the exact same variable, and thus all of the original measurement error would return. Importantly, what we see is that this is a limitation of the data that MO cannot overcome when cell-level priors are directly created from the observed data. As alternate proxies contain correlated errors, identifying the amount of the variance in the proxies by the correlation of the measures is misleading. Positive or negative correlation in the measurement errors leads, respectively, to under- or overestimation of the amount of measurement error in the data, directly biasing results as in EIV. When cell priors are set by the use of auxiliary proxies, our method continues to require the measurement errors (although not the indicators themselves of course) be uncorrelated across alternate measures, so that it is possible to consistently estimate the degree of measurement error present in the data. Again, if there exists additional auxiliary information to set the prior variances, other than the proxies, the MO model has very small bias, as shown in simulations in Figure 7(c), where we assume the true measurement error variance is known from auxiliary information about the measurement procedure.

Even in this most difficult of settings, MO remains robust. In another set of simulations, we compare how various estimators perform when both proxies are correlated with $y_{i}$. Allowing these simulations to vary the amount of correlation gives an indication of how various estimators perform in this difficult situation. ${ }^{10}$ Figure 8 shows that MO outperforms EIV at every level of this correlation. When the dependence between the error and $y_{i}$ is weak, MO almost matches its zero-correlation minimum. Thus, MO appears to be robust to even moderate violations of these assumptions, especially when 


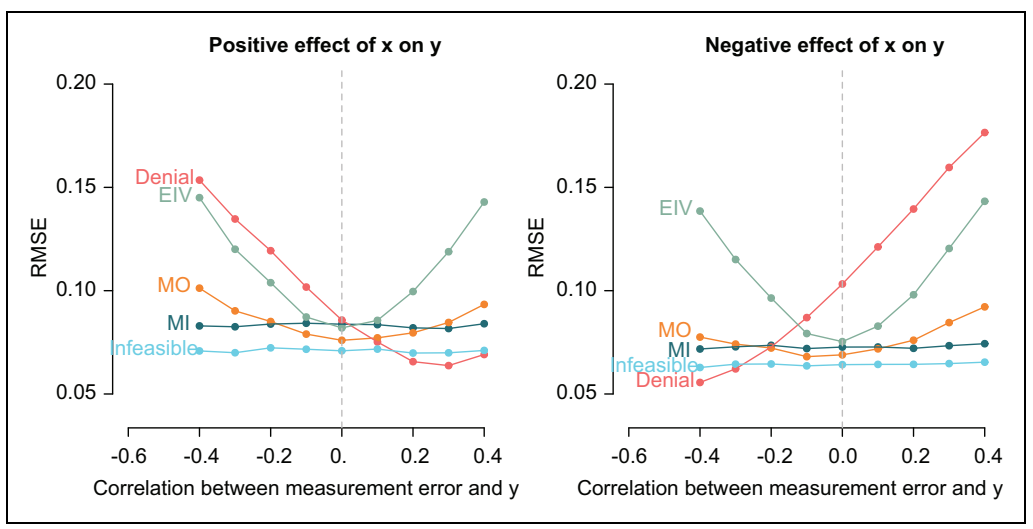

Figure 8. Root mean squared error for various estimators with data generated so that each proxy variable has measurement error correlated with the dependent variable. On the left, $x_{i}$ has a positive relationship with $y_{i}$ and on the right, it has a negative effect. Note that both errors-in-variables (EIV; green) and multiple overimputation (MO; orange) perform worse as the correlation moves away from zero, but $\mathrm{MO}$ always performs better. The denial estimator can actually perform well in certain situations, yet this depends heavily on the direction of the relationship. Both the infeasible estimator and multiple imputation (MI) are unaffected by the amount of correlation.

compared with other measurement error approaches. Interestingly, the denial estimator can perform better than all estimators under certain conditions, yet these conditions depend heavily on the parameters of the data. If we change the effect of $x_{i}$ on $y_{i}$ from negative to positive, the performance of the denial estimator reverses itself. Since we obviously have little knowledge about all of these parameters a priori, the denial estimator is of little use.

Since there are gold standard data in these simulations, we can also investigate the performance of simply discarding the mismeasured data and running MI. As expected, MI is unaffected by the degree of correlation since it disregards the correlated proxies. Yet these proxies have some information when the correlation is around zero and, due to this, MO outperforms MI in this region. As the correlation increases, though, it becomes clear that simply imputing the mismeasured cells has more desirable properties. Of course, with such high correlation, we might wonder if these are actually proxies in our data or simply new variables.

These simulations give key insights into how we should handle data measured with error. MO is appropriate when we have a variable that we can reasonably describe as a proxy - that is, having roughly uncorrelated, mean zero 
Table I. Coverage and MSE for MO by Number of Imputations.

\begin{tabular}{lcccc}
\hline$B$ & $x_{i}$ Coverage & $x_{i}$ MSE & $z_{i}$ Coverage & $z_{i}$ MSE \\
\hline 3 & .931 & .0162 & .936 & .0147 \\
5 & .945 & .0157 & .959 & .0145 \\
10 & .944 & .0152 & .954 & .0135 \\
25 & .945 & .0147 & .957 & .0132 \\
50 & .944 & .0147 & .953 & .0131 \\
100 & .948 & .0146 & .951 & .0131 \\
\hline
\end{tabular}

Note: $\mathrm{MO}=$ multiple overimputation; $\mathrm{MSE}=$ mean square error.

error. Even if these assumptions fail to hold exactly, MO retains its desirable properties. In situations where we suspect that the measurement error on all of our proxies has moderate correlation with other variables in the data, it may be wiser to treat the mismeasurement as pure missingness and use MI. Of course, this approach assumes there exist gold standard data, which may be scarce.

\section{The Number of Data Sets to Overimpute}

MI relies on simulation to reflect the uncertainty due to missing data and requires a sufficient number of such in order to do so accurately. The simulations in this case are the number of imputed data sets. There is a large body of research analyzing the performance of MI as the number of imputed data sets grows, with a general consensus that somewhere between 5 and 50 imputations is sufficient to obtain approximately valid confidence intervals. With $\mathrm{MO}$, we might worry that the increased demands on the imputation model might require additional imputations. In this section, we perform a simulation experiment to see how our uncertainty estimates perform as a function of the number of imputations.

To do so, we use the basic design from Monte Carlo Evidence subsection in BHK, vary the number of imputed data sets, and assess the confidence interval coverage for the estimated coefficients in the outcome model. ${ }^{11}$ We present the results in Table 1, which shows that the qualitative results from MI carry over to MO. In terms of both point estimates and confidence interval coverage, there are only minor improvements beyond 10 to 25 imputed data sets. The lowest number of imputations can lead to confidence intervals that are both too narrow and too wide.

The amount of measurement error in a variable increases the amount of missing information, so we would expect the performance of MO to vary 
Table 2. Coverage for MO at Varying Levels of Proxy Error.

\begin{tabular}{lccccccccc}
\hline & \multicolumn{10}{c}{$\rho$} \\
\cline { 2 - 9 }$B$ & 0.1 & 0.2 & 0.3 & 0.4 & 0.5 & 0.6 & 0.7 & 0.8 & 0.9 \\
\hline 3 & 0.953 & 0.936 & 0.936 & 0.908 & 0.920 & 0.919 & 0.915 & 0.912 & 0.907 \\
5 & 0.953 & 0.942 & 0.951 & 0.955 & 0.953 & 0.961 & 0.965 & 0.965 & 0.977 \\
10 & 0.945 & 0.958 & 0.952 & 0.962 & 0.975 & 0.991 & 0.987 & 0.994 & 0.997 \\
25 & 0.946 & 0.967 & 0.968 & 0.976 & 0.986 & 0.995 & 0.997 & 0.999 & 1.000 \\
50 & 0.952 & 0.958 & 0.970 & 0.978 & 0.987 & 0.999 & 0.999 & 0.999 & 1.000 \\
100 & 0.945 & 0.965 & 0.968 & 0.975 & 0.994 & 0.997 & 1.000 & 1.000 & 1.000 \\
\hline
\end{tabular}

Note: $\mathrm{MO}=$ multiple overimputation.

as a function of the amount of measurement error in the data. It is instructive, then, to investigate the coverage of the MO approach. In Table 2, we display the nominal 95 percent confidence intervals as a function of both $B$ and $\rho$. Here, we are using the same simulation design as in the Specifying or Estimating the Measurement Error Variance section of BHK using the correct value of $\rho$ in the imputation. As is clear from the table, our intuition is correct - the coverage performance of MO weakens as the amount of measurement error increases. In fact, when $\rho>.5$, the confidence intervals become strikingly uninformative. This makes sense considering the fact that less than half of our mismeasured variable's variance is due to the true value. When $\rho$ is at more reasonable values, we see similar results to Table 1 in that the marginal value of increasing $B$ is fairly low after 10 to 25 imputations.

\section{Concluding Remarks and Further Extensions}

This article, taken together with its companion BHK, offers a new approach to preprocessing data so that applied researchers can attend to the serious problems of missing data and measurement error scattered throughout most data sets used in the social sciences. It should be attractive to applied researchers especially since, after attending to these issues, they can apply whatever method they would have in the absence of these problems. This enables social scientists and others to get back to the substantive problems that drove them to analyze their data to begin with.

The methods offered here are implemented in Amelia (Honaker, King, and Blackwell 2010) and can be easily combined with other techniques as part of the same algorithm, most of which we have not been touched on in these two articles. For example, researchers can choose to put prior values 
on individual cell values, include features for time series or time-series cross-sectional data, implement transformations, include many types of prior information, and evaluate results and judge model fit using a wide range of diagnostics.

\section{Authors' Note}

For helpful comments, discussions, and data, we thank Walter Mebane, Gretchen Casper, Simone Dietrich, Justin Grimmer, Sunshine Hillygus, Burt Monroe, Adam Nye, Michael Peress, Eric Plutzer, Mo Tavano, Shawn Treier, Joseph Wright, and Chris Zorn. All code and information needed to replicate this work are available in a Dataverse replication file at Blackwell, Honaker, and King (2015).

\section{Declaration of Conflicting Interests}

The author(s) declared no potential conflicts of interest with respect to the research, authorship, and/or publication of this article.

\section{Funding}

The author(s) disclosed receipt of the following financial support for the research, authorship, and/or publication of this article: This work was supported by the NSF (SES-1059723).

\section{Notes}

1. If a function $f(a, b)$ can be expressed as a family of probability densities for variable $a$ given parameter $b, p(a \mid b)$, and a family of densities for variable $b$ given parameter $a, p(b \mid a)$, so that $f(a, b)=p(a \mid b)=p(b \mid a)$, then $p(a \mid b)$ and $p(b \mid a)$ are said to be statistically dual.

2. If there are missing values in unit $i$, we need to alter the definitions of the distributions to account for values in $x_{i, \text { mis }}$ that have no corresponding $w_{i}$.

3. If it were possible for a measurement error model to work in all contexts, without any auxiliary information to describe the measurement error, the same data set could answer any question!

4. In this simulation, $\beta=(0,1,0.5)$ and $\varepsilon_{i} \sim \mathcal{N}(0,1.5)$. The additional covariate, $z_{i}$, is distributed independent and identically distributed $\left(z_{i} \sim \mathcal{N}(1,1)\right)$. The same size is 1,000 and there are 1,000 simulations.

5. In these simulations, $n=200,\left(x_{i}, y_{i}\right) \sim \mathcal{N}(\mu, \Sigma), \mu=(1,1), \Sigma=(10.4,0.41)$, $\sigma_{u}^{2}=0.5, \sigma_{v}^{2}=0.5$.

6. In a multivariate setting, this becomes $\hat{\delta}=(w 1, w 2)^{-1} w 1, Y$ where $W_{j}$ is the set of regressors using the $j$ th proxy measure for $x$.

7. In these simulations, similar to previous, $n=200,\left(x_{i}, y_{i}, u_{i}, v_{i}\right) \sim \mathcal{N}(\mu, \Sigma)$,

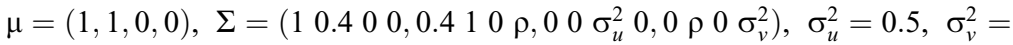


0.5. Thus, the measurement errors are drawn at the same time as $x_{i}$ and $y_{i}$ with mean zero. While $\rho$ allows the error, $v_{i}$, to covary with $y_{i}$, and across the simulations it is set as one of $\rho \in\{-0.3,-0.1,0.1,0.3\}$. The observed mismeasured variables are constructed as $w_{i 1}=x_{i}+u_{i}, w_{i 2}=x_{i}+v_{i}$.

8. Similar to the construction of the last simulations, we set $n=200$,

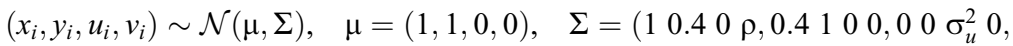
$\left.\rho 00 \sigma_{v}^{2}\right), \sigma_{u}^{2}=0.5, \sigma_{v}^{2}=0.5$, and sequencing $\rho \in\{-0.3,-0.1,0.1,0.3\}$ across sets of simulations.

9. Here we set $n=200,\left(x_{i}, y_{i}, u_{i}, v_{i}\right) \sim \mathcal{N}(\mu, \Sigma), \mu=(1,1,0,0), \Sigma=\left(\begin{array}{llll}1 & 0.4 & 0 & 0\end{array}\right.$, $\left.0.4100,00 \sigma_{u}^{2} \rho, 00 \rho \sigma_{v}^{2}\right), \sigma_{u}^{2}=0.5, \sigma_{v}^{2}=0.5$, and sequencing $\rho \in\{-0.3$, $-0.1,0.1,0.3\}$ across sets of simulations.

10. These simulations follow the abovementioned pattern except they include a perfectly measured covariate, $z_{i}$, which determines which observations are selected for mismeasurement. Thus, we have $\left(x_{i}, y_{i}, z_{i}, u_{i}, v_{i},\right) \sim N(\mu, \Sigma)$, with $\mu=(1,1,-1,0,0)$ and $\Sigma=\left(\begin{array}{ll}1 \sigma_{x y}-0.400, \sigma_{x y} 1-0.2 \rho \sigma_{u} \rho \sigma_{v},-0.4 & -1\end{array}\right.$ $-0.21000 \rho \sigma_{u} 0 \sigma_{u}^{2} 0,0 \rho \sigma_{v} 00 \sigma_{v}^{2}$ ) with $\sigma_{u}^{2}=0.5$ and $\sigma_{v}^{2}=0.75$. We ran simulations at both $\sigma_{x y}=0.4$ and $\sigma_{x y}=-0.4$. Each observation had probability $\pi_{i}=\left(1+\mathrm{e}^{3.5+2 z}\right)^{-1}$, which has a mean of .25 . We used the multiple proxies approach to estimating the measurement error. For errors-in-variables, we applied the model as if the entire variable were mismeasured.

11. Here, we set the assumed amount of measurement error to the correct level.

\section{References}

Bityukov, S. I., V. V. Smirnova, N. V. Krasnikov, and V. A. Taperechkina. 2006. "Statistically Dual Distributions in Statistical Inference." Pp. 102-5 in Statistical Problems in Particle Physics, Astrophysics and Cosmology: Proceedings of PHYSTAT05, Oxford, UK, 12-15 September 2005. Retrieved April 1, 2015. http:// arxiv.org/abs/math/0411462v2.

Blackwell, Matthew, James Honaker, and Gary King. 2015. "Replication Data for A Unified Approach to Measurement Error and Missing Data: Details and Extensions." Retrieved April 1, 2015. (http://dx/doi.org/10.7910/DVN/29610. IQSS Dataverse Network [Distributor]).

Blackwell, Matthew, James Honaker, and Gary King. 2017. "A Unified Approach to Measurement Error and Missing Data: Overview." Sociological Methods and Research 46: 303-41.

Honaker, James and Gary King. 2010. "What to Do About Missing Values in Time Series Cross-section Data.” American Journal of Political Science 54:561-81. Retrieved April 1, 2015. (http://gking.harvard.edu/files/abs/pr-abs.shtml).

Honaker, James, Gary King, and Matthew Blackwell. 2010. “Amelia II: A Program for Missing Data.” Retrieved April 1, 2015. (http://gking.harvard.edu/amelia). 
Katz, Jonathan N. and Gabriel Katz. 2010. "Correcting for Survey Misreports Using Auxiliary Information with an Application to Estimating Turnout." American Journal of Political Science 54:815-35.

King, Gary, James Honaker, Anne Joseph, and Kenneth Scheve. 2001. “Analyzing Incomplete Political Science Data: An Alternative Algorithm for Multiple Imputation." American Political Science Review 95:49-69. Retrieved April 1, 2015. (http://gking.harvard.edu/files/abs/evil-abs.shtml).

Newey, Whitney K. and Daniel McFadden. 1994. "Large Sample Estimation and Hypothesis Testing." Pp. 2111-245 in Handbook of Econometrics, edited by Robert F. Engle and Daniel L. McFadden. Amsterdam, the Netherlands: Elsevier. Schafer, Joseph L. 1997. Analysis of Incomplete Multivariate Data. London, UK: Chapman \& Hall.

Schafer, Joseph L. and Maren K. Olsen. 1998. "Multiple Imputation for Multivariate Missing-data Problems: A Data Analyst's Perspective." Multivariate Behavioral Research 33:545-71.

Seaman, Shaun, John Galati, Dan Jackson, and John Carlin. 2013. "What Is Meant by "Missing at Random"?" Statistical Science 28:257-68.

Stefanski, L. A. 2000. "Measurement Error Models." Journal of the American Statistical Association 95:1353-58.

Wu, C. F. Jeff. 1983. "On the Convergence Properties of the EM Algorithm." The Annals of Statistics 11:95-103.

\section{Author Biographies}

Matthew Blackwell is an assistant professor in the Department of Government at Harvard University.

James Honaker is a senior research scientist in the Institute for Quantitative Social Science at Harvard University.

Gary King is the Albert J. Weatherhead III university professor and director of the Institute for Quantitative Social Science at Harvard University. For more information, see GaryKing.org. 